\title{
Prognosis and Clinical Characteristics of Patients with Pancreatic Ductal Adenocarcinoma Diagnosed by Endoscopic Ultrasonography but Indeterminate on Computed Tomography
}

\author{
Sung Woo Ko ${ }^{1 *}$, Tae Hyeon $\mathrm{Kim}^{2}$, Tae Jun Song ${ }^{1}$, Seong-Hun Kim³ , Dong-Wan Seo ${ }^{1}$, Jai Hoon Yoon ${ }^{4}$, Chang \\ Min $\mathrm{Cho}^{5}$, Jae Hee Cho ${ }^{6}$, Jun-Ho Choi ${ }^{7}$, Dong Wook Lee ${ }^{8 \dagger}$, Sang Hyub Lee ${ }^{9}$, Seung Bae Yoon ${ }^{10}$, Tae Hoon Lee ${ }^{11}$, \\ Gwang Ha Kim ${ }^{12}$, and Hoon Jai Chun ${ }^{13}$ \\ ${ }^{1}$ Department of Gastroenterology, Asan Medical Center, Seoul, ${ }^{2}$ Department of Internal Medicine, Wonkwang University School of \\ Medicine, Iksan, ${ }^{3}$ Department of Internal Medicine, Research Institute of Clinical Medicine of Jeonbuk National University-Biomedical \\ Research Institute of Jeonbuk National University Hospital, Jeonju, ${ }^{4}$ Division of Gastroenterology, Department of Internal Medicine, \\ Hanyang University College of Medicine, Seoul, ${ }^{5}$ Department of Internal Medicine, School of Medicine, Kyungpook National University, \\ Daegu, ${ }^{6}$ Department of Internal Medicine, Gachon University College of Medicine, Incheon, ${ }^{7}$ Department of Internal Medicine, \\ Dankook University College of Medicine, Cheonan, ${ }^{8}$ Department of Internal Medicine, Daegu Catholic University School of Medicine, \\ Daegu, ${ }^{9}$ Department of Internal Medicine and Liver Research Institute, Seoul National University Hospital, Seoul National University \\ College of Medicine, ${ }^{10}$ Department of Internal Medicine, College of Medicine, The Catholic University of Korea, Seoul, ${ }^{11}$ Division of \\ Gastroenterology, Department of Internal Medicine, Soonchunhyang University Cheonan Hospital, Soonchunhyang University School \\ of Medicine, Cheonan, ${ }^{12}$ Department of Internal Medicine, Pusan National University School of Medicine and Biomedical Research \\ Institute, Pusan National University Hospital, Busan, and ${ }^{13}$ Division of Gastroenterology and Hepatology, Department of Internal \\ Medicine, Institute of Digestive Disease and Nutrition, Korea University College of Medicine, Seoul, Korea
}

\author{
Article Info \\ Received March 18, 2021 \\ Revised July 1, 2021 \\ Accepted July 13, 2021 \\ Published online October 7, 2021

\section{Corresponding Author} \\ Tae Hyeon Kim \\ ORCID https://orcid.org/0000-0002-9723-2136 \\ E-mail kth@wku.ac.kr \\ Tae Jun Song \\ ORCID https://orcid.org/0000-0002-6156-8746 \\ E-mail medi01@naver.com \\ *Current affiliation of Sung Woo Ko is \\ Department of Internal Medicine, Eunpyeong \\ St. Mary's Hospital, College of Medicine, The \\ Catholic University of Korea, Seoul, Korea \\ ${ }^{\dagger}$ Current affiliation of Dong Wook Lee is \\ Department of Internal Medicine, School of \\ Medicine, Kyungpook National University, \\ Daegu, Korea.
}

Background/Aims: Endoscopic ultrasonography (EUS) provides high-resolution images and is superior to computed tomography (CT) scan in diagnosing small pancreatic ductal adenocarcinoma (PDAC). As a result, the use of EUS for early detection of PDAC has attracted attention. This study aimed to identify the clinical and radiological characteristics of patients with PDAC diagnosed by EUS but not found on CT scan.

Methods: The medical records of patients diagnosed with PDAC at 12 tertiary referral centers in Korea from January 2003 to April 2019 were reviewed. This study included patients with pancreatic masses not clearly observed on CT scan but identified on EUS. The clinical characteristics and radiological features of the patients were analyzed, and survival analysis was performed.

Results: A total of 83 patients were enrolled. The most common abnormal CT findings other than a definite mass was pancreatic duct dilatation, which was identified in 61 patients $(73.5 \%)$. All but four patients underwent surgery. The final pathologic stages were as follows: IA ( $n=31,39.2 \%)$, IB $(n=8,10.1 \%)$, IIA $(n=20,25.3 \%)$, IIB $(n=17,21.5 \%)$, III $(n=2,2.5 \%)$, and IV $(n=1,1.4 \%)$. The 5 -year survival rate of these patients was $50.6 \%$ (95\% confidence interval, $38.8 \%$ to $66.7 \%$ ). Elevated liver function testing and R1 resection emerged as significant predictors of mortality in the multivariable Cox regression analysis.

Conclusions: This multicenter study demonstrated favorable long-term prognosis in patients with PDAC diagnosed by EUS but indeterminate on CT scan. EUS should be considered for patients with suspected PDAC but indeterminate on CT scan. (Gut Liver 2022;16:474-482)

Key Words: Pancreatic neoplasms; Endosonography; Kaplan-Meier estimate
INTRODUCTION

Despite recent advances in surgical techniques and chemotherapeutic agents, the prognosis of pancreatic ductal adenocarcinoma (PDAC) is still poor with a 5-year survival rate of $9.3 \%$ according to the National Cancer 
Institute of the United States. ${ }^{1}$ This poor prognosis may be attributed to difficulty in early detection as most patients are asymptomatic in the early stage, and there is no reliable biomarker for PDAC. ${ }^{2}$ As a result, only around $20 \%$ of patients are eligible for surgical resection at the time of diagnosis. ${ }^{3}$ Unlike gastric or colorectal cancer, the lack of an effective screening method for PDAC is one of the reasons that make early detection difficult. However, there are several reports indicating that a favorable prognosis can be expected when PDAC is detected in the early stage. In the case of PDAC confined to the pancreatic parenchyma, the 5-year survival rate has been reported to be $34.3 \%{ }^{1}$ The 5 -year survival rate of patients with PDAC smaller than 1 $\mathrm{cm}$ is up to $80.4 \%$ according to the Japan Pancreatic Cancer Registry. Therefore, early detection of PDAC is crucial to improve prognosis.

Recently, endoscopic ultrasonography (EUS) has attracted attention with its high sensitivity for detecting tumors of the pancreas. In a review of 22 studies to compare imaging modalities for the detection of pancreatic tumors, the sensitivity of computed tomography (CT) was $74 \%$, whereas the sensitivity of EUS was up to $98 \%{ }^{5}$ As EUS can provide high-resolution images, it can be useful particularly when the tumor is small. ${ }^{6}$ For pancreatic tumors smaller than $3 \mathrm{~cm}$, the sensitivity of EUS, CT, and magnetic resonance imaging has been reported as $93 \%, 53 \%$, and $67 \%$, respectively. ${ }^{7}$ In clinical practice, it is not uncommon to detect small pancreatic tumors on EUS, which had not been identified on other imaging modalities (Fig. 1).

Thus far, the clinical characteristics, pathological stage after surgery, and long-term prognosis of patients with PDAC diagnosed by EUS but indeterminate on CT have not been well established. The purpose of this study was to identify the aforementioned parameters in patients with PDAC diagnosed by EUS but indeterminate on CT.

\section{MATERIALS AND METHODS}

\section{Patients}

Between January 2003 and April 2019, the medical records of patients with PDAC diagnosed by EUS but indeterminate on CT from 12 tertiary referral hospitals in South Korea were reviewed retrospectively. The inclusion criteria were as follows: (1) age $\geq 18$ years; (2) pancreatic mass identified on EUS but indeterminate on CT; or (3) histopathological diagnosis was established by EUS-guided fine-needle aspiration (EUS-FNA) or surgically resected specimens. The exclusion criteria were as follows: (1) patients with pancreatic mass clearly identified on CT; (2) patients whose histopathological diagnosis was not confirmed; or (3) patients with pancreatic neoplasms other than ductal adenocarcinoma, such as neuroendocrine tumor or lymphoma. Data including the patient's baseline demographics, laboratory findings, pathological stages after surgery, and follow-up monitoring were also recorded. The protocol was approved by the institutional review board of each hospital enrolled in the study (Asan Medical Center IRB approval number: S2019-1839-0004). The need for informed consent was waived.

\section{EUS technique}

EUS was performed using a radial (GF-UM2000, GFUE260-AL5; Olympus Medical, Tokyo, Japan) or linear array echoendoscopes (GF-UCT240-AL5, GF-UCT260; Olympus Medical) for detecting the pancreatic mass. All EUS-FNA procedures were performed using a linear array echoendoscope connected to a processor featuring color Doppler function (ProSound Alpha 5 or Alpha 10; Hitachi Aloka Medical, Ltd., Tokyo, Japan or UM-ME1 or UMME2; Olympus Medical). Experienced endosonographers in each hospital performed all procedures with conscious sedation (with midazolam or propofol combined with
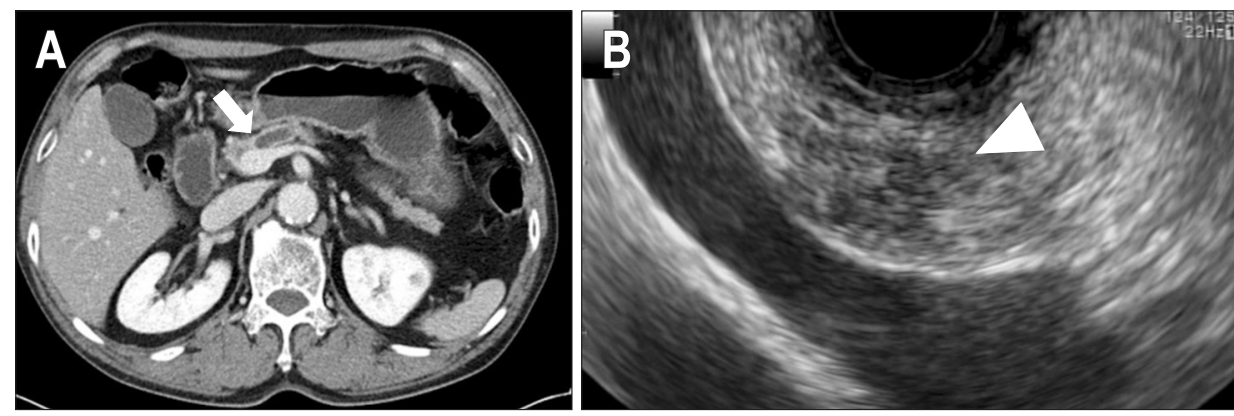

Fig. 1. A 66-year-old male underwent an abdomen computed tomography (CT) scan for a medical checkup. (A) The CT scan showed diffuse pancreatic duct dilatation, but no mass-like lesions were identified in the pancreas (white arrow). (B) On endoscopic ultrasonography, an approximately 6-mm-sized hypoechoic mass was identified in the body of the pancreas (white arrowhead). Fine-needle aspiration confirmed it to be adenocarcinoma. Distal pancreatectomy was performed, and the pathological stage was IA. The patient was followed up over a period of 6 years without recurrence. 
pethidine). Once masses were identified on EUS, the largest diameter was measured. When EUS-FNA was performed, the type and size of EUS-FNA needles were determined according to the endosonographer's preference in each hospital. Contrast-enhanced EUS and rapid on-site evaluation were not used in all cases in the study cohort.

\section{CT protocol and definition of CT findings}

Before EUS, CT was performed using a 64- or 256-detector-row scanner. After the patients were given contrast media intravenously, 3 phases of abdominal images (unenhanced, arterial, portal) were acquired with a standard CT protocol in each hospital. Section thickness was 2.5 $\mathrm{mm}$ or $5 \mathrm{~mm}$. Indeterminate CT findings was defined that there were ancillary findings but no definitive mass in the pancreas. The definition of each indeterminate CT finding was as follows: (1) focal alteration of pancreatic parenchymal attenuation: localized tissue enhancement that differs from the surrounding pancreatic parenchyma described by the radiologists; (2) bile duct dilatation: diameter of the common bile duct greater than $8 \mathrm{~mm}$; (3) pancreatic duct dilatation: diameter of the main pancreatic duct greater than $3 \mathrm{~mm}, 2 \mathrm{~mm}$, and $1 \mathrm{~mm}$ in the head, body, and tail of the pancreas, respectively; (4) double-duct sign: combined dilatation of the common bile duct and pancreatic duct; or (5) peripancreatic lymphadenopathy: enlarged lymph nodes around the pancreas by $1 \mathrm{~cm}$ or more.

\section{Staging workup}

To determine the extent of the disease, staging workup was performed for all patients. Positron emission tomography-CT was performed in all but one patient, and chest$\mathrm{CT}$ was performed in 13 patients. All patients underwent at least one of positron emission tomography-CT or chestCT for staging workup.

\section{Outcomes}

The baseline characteristics of the patient population, pancreatic tumors, and procedural details were analyzed. The primary outcome was overall survival (defined as the time from date of PDAC diagnosis to date of death from any cause) for all cohorts. The secondary outcomes were progression-free survival (defined as the time from date of PDAC diagnosis to earliest disease progression or death), pathological staging based on surgically resected specimen according to the American Joint Committee on Cancer staging manual 7th edition, abnormal findings detected by $\mathrm{CT}$, and the findings of EUS and EUS-FNA for all enrolled patients.

\section{Statistical analysis}

Descriptive statistics such as the mean, median, standard deviation, and interquartile range were calculated to summarize continuous variables. The sensitivity of EUSFNA for diagnosing PDAC was also quantified. Survival analysis was performed using the Kaplan-Meier method. Multivariable Cox regression was performed to identify factors associated with overall survival. Using a backward elimination, variables with $\mathrm{p}$-value $<0.2$ on univariable regression were included as covariates for multivariable Cox regression. The results of the Cox regression were presented as a hazard ratio with corresponding $95 \%$ confidence interval (CI). Statistical significance was determined as $\mathrm{p}<0.05$. All statistical analyses were performed using $\mathrm{R}$ software version 3.5.3 (R Foundation for Statistical Computing, Vienna, Austria; http://www.R-project.org).

\section{RESULTS}

\section{Patients' characteristics}

From January 2003 to April 2019, 83 patients who met the inclusion criteria were identified. The mean age was 64.2 years $( \pm 10.9)$, and 46 patients $(55.4 \%)$ were male. In addition, 17 patients $(20.5 \%)$ had a history of pancreatitis, and three patients (3.4\%) had a family history of PDAC.

Indications for abdominal CT were as follows: abdominal pain ( $\mathrm{n}=47,56.7 \%)$, medical checkup ( $\mathrm{n}=17,20.5 \%)$, jaundice ( $\mathrm{n}=11,13.3 \%)$, weight loss $(\mathrm{n}=2,2.4 \%)$, and fever $(\mathrm{n}=1,1.2 \%)$. Another five patients $(5.9 \%)$ showed abnormal CT findings during the surveillance of diseases in other organs. Elevated liver function (define as three or more positive parameters including bilirubin, alkaline phosphatase, gamma-glutamyl transpeptidase, alanine transaminase, and aspartate transaminase) and carbohydrate antigen 19-9 (CA19-9) level ( $\geq 37 \mathrm{U} / \mathrm{mL})$ were noted in 21 patients (25.3\%) and 25 patients (30.1\%), respectively.

Abnormal findings detected by CT are summarized in Table 1. The most common abnormal finding was pancreatic duct dilatation, which was identified in 61 patients (73.5\%). Other findings were focal alteration of paren-

Table 1. Abnormal Findings on Abdominal Computed Tomography

\begin{tabular}{lc}
\hline \multicolumn{1}{c}{ Computed tomography findings } & No. of patients $(\%)$ \\
\hline Pancreatic duct dilatation & $61(73.5)$ \\
Focal alteration of parenchymal attenuation & $14(16.9)$ \\
Double-duct sign & $10(12.0)$ \\
Bile duct dilatation & $9(10.8)$ \\
Metastatic lymphadenopathy & $8(9.6)$ \\
Splenic infarction & $1(1.2)$ \\
Normal & $1(1.2)$ \\
\hline
\end{tabular}


chymal attenuation in 14 patients, double-duct sign in 10 patients, bile duct dilatation in nine patients, metastatic lymphadenopathy in eight patients, and splenic infarction in one patient. All lymph nodes identified on CT were regional lymphadenopathy (e.g., lymph nodes in hepatoduodenal ligament) expected to be curatively resected. One patient underwent CT for workup for a pancreatic mass found by transabdominal ultrasonography during medical checkup; however, no abnormal findings were noted on the CT scan. The patient then underwent a EUS examination, and the mass was identified.

\section{EUS findings}

In all patients who underwent EUS, the pancreatic mass was identified on EUS. The location of pancreatic mass on EUS was as follows: head/uncinate process in 45 patients (54.2\%), body in 33 patients (39.8\%), and tail in five patients (6\%). The mean size of the masses was $15.3 \pm 4.6 \mathrm{~mm}$ in the greatest dimension, and most of the masses were smaller than $20 \mathrm{~mm}$ ( $\mathrm{n}=75,90.4 \%)$.

EUS-FNA was performed in 71 patients (85.5\%). For EUS-FNA, 22-gauge needles were used in 47 patients (66.2\%), and 25-gauge needles were used in 21 patients $(29.6 \%)$, and a 19 -gauge needle in one patient (4.2\%). Among the 71 patients, EUS-FNA revealed definite ductal adenocarcinoma in 45 patients (63.4\%) and atypical cells suspicious for adenocarcinoma in 15 patients (21.1\%). The remaining 11 patients did not reveal malignancy. As a result, the pooled sensitivity of EUS-FNA for detecting PDAC in patients with indeterminate abdominal CT findings was $84.5 \%$. The specificity, positive predictive value, and negative predictive value were not calculated because only patients confirmed as having PDAC were included in this study. The findings of EUS and EUS-FNA are summarized in Table 2. Magnetic resonance cholangiopancreatography was performed in 66 patients (79.5\%). Among them,

Table 2. Findings of EUS, EUS-FNA

\begin{tabular}{lc}
\hline \multicolumn{1}{c}{ Finding } & No. of patients (\%) \\
\hline Mass location on EUS & \\
Head/uncinate process & $45(54.2)$ \\
Body & $33(39.8)$ \\
Tail & $5(6.0)$ \\
Size of mass & \\
$\quad$ Mean $\pm S D, m m$ & $15.3 \pm 4.6$ \\
$\leq 20.0 \mathrm{~mm}$ & $75(90.4)$ \\
EUS-FNA & $71(85.5)$ \\
Adenocarcinoma & $45(63.3)$ \\
Atypical cells, suspicious for adenocarcinoma & $15(21.1)$ \\
Inflammation & $4(4.8)$ \\
Inadequate sample & $7(10.8)$ \\
\hline
\end{tabular}

EUS, endoscopic ultrasonography; EUS-FNA, EUS-guided fineneedle aspiration. the mass was identified in 46 patients (69.7\%) on magnetic resonance cholangiopancreatography.

\section{Pathological staging and survival analysis}

Of the 83 patients, 79 patients underwent surgical resection for PDAC. The remaining four patients either refused surgery $(\mathrm{n}=2)$ or had a medical condition that was unfit for surgery $(\mathrm{n}=2)$. Two patients received neoadjuvant chemotherapy before surgery. Pancreaticoduodenectomy or pylorus-preserving pancreaticoduodenectomy was performed in 49 patients, and 30 patients underwent distal pancreatectomy. R0 resection was achieved in 63 patients (79.7\%). The pathological staging of surgically resected specimens was performed according to the American Joint Committee on Cancer staging manual 7 th edition. ${ }^{6}$ Sixty patients (75.9\%) showed no lymph node metastasis. The postoperative pathological stages were as follows: IA $(n=31$, $39.2 \%)$, IB ( $n=8,10.1 \%)$, IIA ( $n=20,25.3 \%)$, IIB $(n=17$, $21.5 \%)$, III ( $\mathrm{n}=2,2.5 \%)$, and IV ( $\mathrm{n}=1,1.4 \%)$ (Table 3). In addition, 41 patients received adjuvant chemotherapy after surgery.

The median duration of follow-up was 22.7 months (interquartile range, 13.7 to 52.8 ) in the study cohort. The overall 1-year and 5-year survival rate was $93.4 \%$ (95\% CI, $88 \%$ to $99.2 \%$ ) and $50.6 \%$ (95\% CI, $38.8 \%$ to $66.7 \%$ ), respectively (Fig. 2A). The overall 1-year and 5-year recurrence-free survival rate was $70.3 \%$ (95\% CI, $60.6 \%$ to $81.6 \%$ ) and $36.6 \%$ (95\% CI, $23.6 \%$ to $48.2 \%$ ), respectively. We also performed a multivariate Cox regression analysis to identity variables relevant to survival. Age, sex, elevated liver function test (LFT), elevated CA19-9, tumor location,

Table 3. pTNM Staging According to the American Joint Committee on Cancer 7th Edition

\begin{tabular}{|c|c|}
\hline & No. of patients (\%) \\
\hline \multicolumn{2}{|c|}{ T stage } \\
\hline $\mathrm{T} 1$ & 33 (41.7) \\
\hline $\mathrm{T} 2$ & $9(11.4)$ \\
\hline T3 & $36(45.6)$ \\
\hline T4 & $1(1.4)$ \\
\hline \multicolumn{2}{|c|}{$\mathrm{N}$ stage } \\
\hline No & 60 (75.9) \\
\hline N1 & $19(24.1)$ \\
\hline \multicolumn{2}{|c|}{ M stage } \\
\hline MO & 78 (98.3) \\
\hline M1 & $1(1.4)$ \\
\hline \multicolumn{2}{|c|}{ TNM stage } \\
\hline IA & 31 (39.2) \\
\hline IB & $8(10.1)$ \\
\hline$\| \mathrm{A}$ & 20 (25.3) \\
\hline IIB & $17(21.5)$ \\
\hline III & $2(2.5)$ \\
\hline IV & $1(1.4)$ \\
\hline
\end{tabular}


A

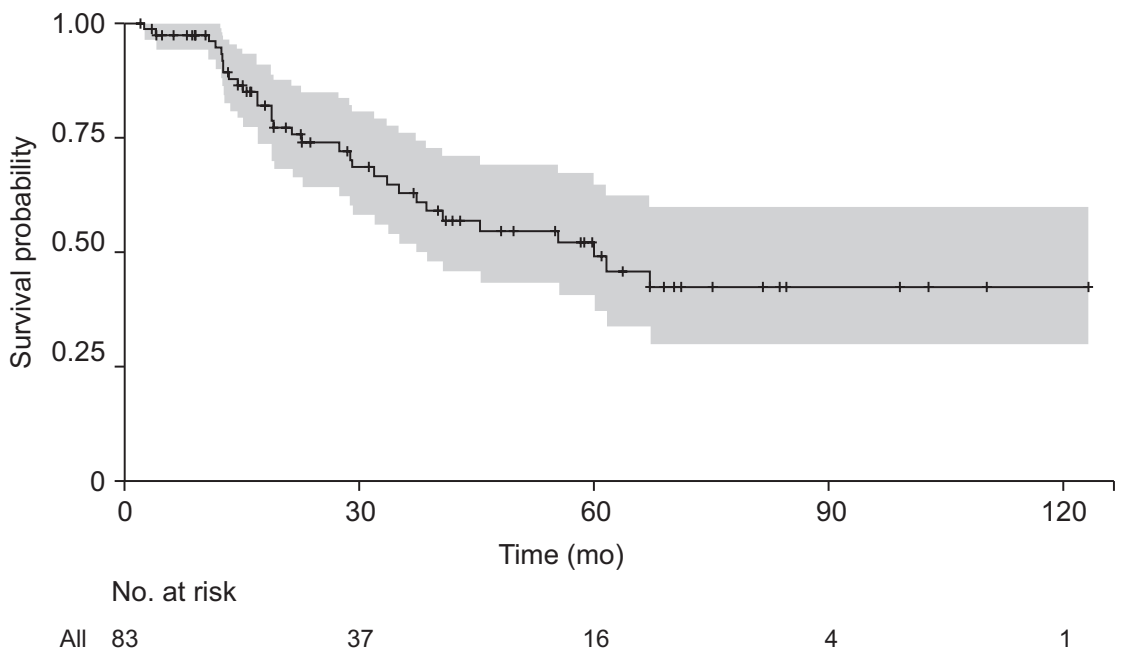

B

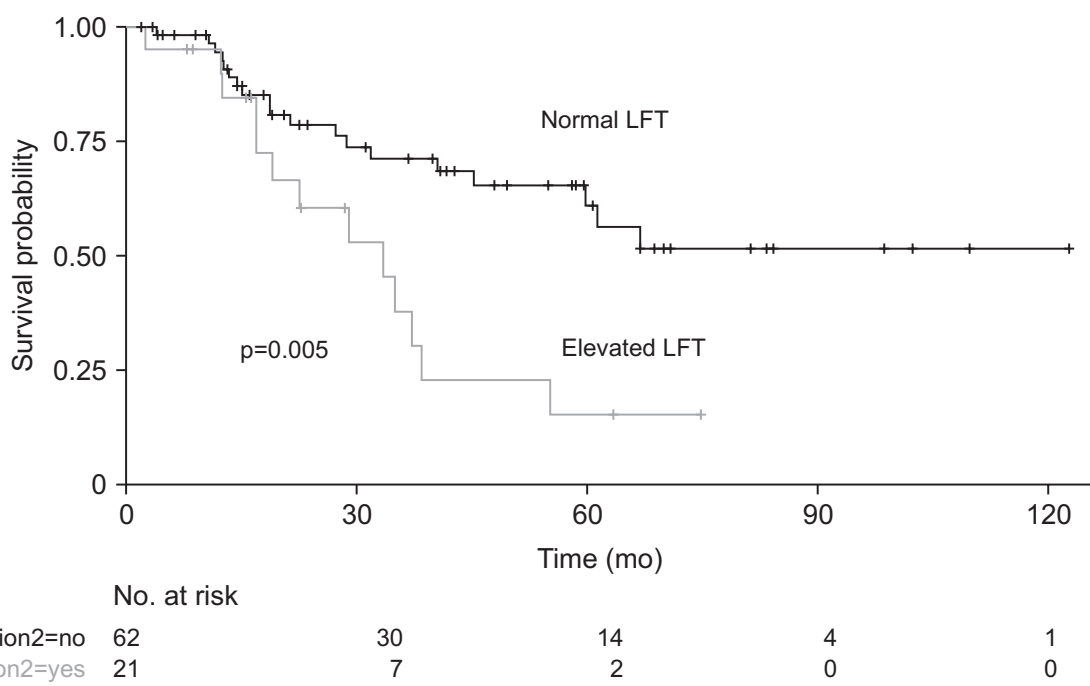

Fig. 2. (A) The Kaplan-Meier curve of patients with pancreatic ductal adenocarcinoma diagnosed by endoscopic ultrasonography but indeterminate on computed tomography. Over a median follow-up of 22.7 months (interquartile range, 13.7 to 52.8 months), the overall 5 -year survival rate was $50.6 \%$ (95\% confidence interval, 38.8 to 66.7 ). (B) The Kaplan-Meier curve of patients according to liver function test (LFT). An elevated LFT result was associated with a lower 5 -year survival rate ( $15.4 \%$ vs $60.6 \%$, log-rank $p=0.005)$.

tumor size, pathologic staging, R1 resection, and lymph node metastasis assessed as potential variables. On univariable Cox regression, elevated LFT, elevated CA19-9, tumor location, pathological staging and R1 resection were found to be eligible for further analysis. Application of multivariate Cox regression analysis using backward elimination demonstrated elevated LFT (hazard ratio, 2.25; 95\% CI, 1.07 to $4.47, \mathrm{p}=0.031$ ) and $\mathrm{R} 1$ resection (hazard ratio, 2.68; $95 \%$ CI, 1.24 to $5.76, \mathrm{p}=0.01$ ) were significant predictors of poorer survival in the final model. The results of the Cox regression analysis are summarized in Table 4. Subgroup analysis of overall survival by LFT was performed by the Kaplan-Meier curve. The patients with elevated LFT had a 5 -year survival rate of $15.4 \%$ ( $95 \% \mathrm{CI}, 4.41 \%$ to $54.1 \%$ ), whereas the patient with normal had a rate of $60.6 \%(95 \%$ $\mathrm{CI}, 46.8 \%$ to $78.5 \%$ ). The difference in overall survival between two groups was significant by log-rank test $(\mathrm{p}=0.005)$ (Fig. 2B).

\section{DISCUSSION}

Our study examined the pathological stage and prognosis of patients with PDAC detected by EUS but indeterminate on CT as well as their clinical characteristics.

CT provides wide anatomic coverage, allowing the lo- 
Table 4. Univariable and Multivariable Cox Regression Model for Overall Survival

\begin{tabular}{|c|c|c|c|c|c|c|}
\hline \multirow{2}{*}{ Variable } & \multicolumn{3}{|c|}{ Univariable analysis } & \multicolumn{3}{|c|}{ Multivariable analysis } \\
\hline & Hazard ratio & $95 \% \mathrm{Cl}$ & $\mathrm{p}$-value & Hazard ratio & $95 \% \mathrm{Cl}$ & $\mathrm{p}$-value \\
\hline Sex (male) & 1.15 & $0.56-2.35$ & 0.702 & & & \\
\hline Age ( $\geq 65 \mathrm{yr})$ & 0.81 & $0.62-2.48$ & 0.549 & & & \\
\hline Elevated liver function test & 2.70 & $1.32-5.53$ & 0.006 & 2.25 & $1.07-4.47$ & 0.031 \\
\hline Elevated CA19-9 & 2.31 & $1.06-5.01$ & 0.034 & & & \\
\hline \multicolumn{7}{|l|}{ Tumor location } \\
\hline Head, uncinated process & 2.06 & $0.96-4.41$ & 0.063 & & & \\
\hline Tumor size $(\geq 2 \mathrm{~cm})$ & 1.39 & $0.63-3.11$ & 0.417 & & & \\
\hline \multicolumn{7}{|l|}{ Pathological staging } \\
\hline Stage IB, II, III, IV vs IA & 2.11 & $0.95-4.71$ & 0.068 & & & \\
\hline $\mathrm{R} 1$ resection & 3.15 & $1.50-6.61$ & 0.002 & 2.68 & $1.24-5.76$ & 0.01 \\
\hline Positive lymph node metastasis & 1.60 & $0.74-3.48$ & 0.234 & & & \\
\hline
\end{tabular}

$\mathrm{Cl}$, confidence interval; CA19-9, carbohydrate antigen 19-9.

cal assessment of tumors (such as vascular involvement) and the evaluation of distant metastasis in a single session. ${ }^{8}$ Due to these advantages, CT is the most widely used imaging modality for diagnosing PDAC, ${ }^{9}$ and the National Comprehensive Cancer Network guidelines recommend $\mathrm{CT}$ as the first choice of imaging modality for patients with clinically suspected PDAC. ${ }^{10}$ However, some tumors are not detected by CT, especially when the size of the tumors is smaller than $2 \mathrm{~cm}^{11,12}$ In a review of 19 comparative studies covering 895 patients, the sensitivity of CT for the detection of pancreatic masses was $53 \%$ to $92 \%$ (pooled sensitivity, $74 \%$ ), and the sensitivity of EUS was $87 \%$ to $100 \%$ (pooled sensitivity, 98\%). ${ }^{5}$ As EUS provides highresolution images, it can be more advantageous when the tumor is small. For the detection of pancreatic tumors smaller than $2 \mathrm{~cm}$, EUS has been reported to have superior sensitivity compared with CT $(94.4 \%$ and $50 \%$, respectively). ${ }^{13}$ Furthermore, given that EUS has a high negative predictive value for PDAC, if masses are not detected by EUS, the possibility of PDAC may be excluded. ${ }^{14}$ Accordingly, the Japan Pancreas Society has recommended EUS as one of the diagnostic tools for patients with suspected PDAC. ${ }^{15}$

The superior sensitivity of EUS in the detection of pancreatic tumors has been demonstrated consistently. ${ }^{16-18}$ All these studies were single-center studies, and most studies included patients with pancreatic tumors other than PDAC and did not define indeterminate CT findings suggesting PDAC. Therefore, it would be clinically significant to identify the characteristics of PDAC detected by EUS but indeterminate on CT. Moreover, the pathological stage after surgery and long-term prognosis and predictors of survival in those patients was not established by previous studies.

In our study, we enrolled only patients with confirmed PDAC from 12 tertiary referral centers in Korea. Pancreatic duct dilatation was most common finding on CT when no definite mass was identified but other abnormal findings were present, which was observed in 71 patients (85.5\%) (including double-duct sign). Studies have been conducted to compare the diagnostic performance of CT and EUS in detecting pancreatic tumors; however, few have reported CT findings suggesting pancreatic tumors. In the study by Wang et al., ${ }^{18}$ multivariate analysis identified pancreatic duct dilatation as an independent risk factor in the detection of PDAC by EUS (odds ratio, 7.24; $95 \%$ CI, 1.97 to 26.7). Several studies have demonstrated that dilatation of pancreatic duct has a high probability of being PDAC. ${ }^{19-21}$ Recently, Fujisawa et al. ${ }^{22}$ investigated the incidence of pancreatic duct dilatation and its relationship with PDAC for healthy population who underwent medical checkups. Dilatation of pancreatic duct was predictive factor for PDAC with odds ratio of 12.684 (95\% CI, 1.70 to $94.48)$ in the logistic regression. This finding is in agreement with the result of our study. Therefore, EUS must be considered for patients suspected to have PDAC but with indeterminate CT findings, especially for those who show pancreatic duct dilatation.

Data regarding the distribution of pathological stages after surgery and long-term prognosis of patients with PDAC diagnosed by EUS but indeterminate on CT are limited. All but four patients in our study underwent pancreatic resection. The pathological stages of the surgically resected specimens from 79 patients was determined; around half of them were classified as stage IA or IB ( $n=39$, $49.3 \%$ ). More notably, around $75 \%$ of the enrolled patients had negative peripancreatic lymph nodes. Over a median follow-up of 22.7 months, the 5-year survival rate was $50.6 \%$ (95\% CI, 38.8 to 66.7 ), which was superior to the survival rate of PDAC recently reported by the Surveillance, Epidemiology, and End Results Program of the National Cancer Institute (9.3\%). ${ }^{1}$ Around half of our patients were classified as stage I, and many of them did not have lymph node metastasis. Lymph node metastasis is a well- 
established negative predictor of survival in patients with PDAC who underwent surgery. In comparison with patients without metastasis, patients with lymph node metastasis after surgery have been found to have a worse survival outcome. $^{23,24}$ Therefore, in the present study, early detection of tumors by EUS may have contributed to a favorable clinical outcome.

In this study cohort, elevated LFT and R1 resection were identified as significant prognostic factors of survival on a multivariable Cox regression. R1 resection is a wellestablished poor prognostic factor after PDAC surgery; ${ }^{25}$ however, the role of elevated LFT as a prognostic factor in patients with PDAC is rarely investigated yet. In the present study, the locations of the masses in the patients with elevated LFT were pancreatic head or uncinate process, the proportion corresponding to stage IA was significantly lower than that of patients with normal LFT $(11.01 \%$ vs $56.9 \%, \mathrm{p}=0.013$ ). However, elevated LFT was still a significant predictor of survival after adjusting potential confounding factors by multivariable Cox regression. Darnell et al. $^{26}$ reported preoperative cholangitis was a risk factor for mortality after pancreaticoduodenectomy. While all patients in the study by Darnell et al. were borderline resectable PDAC who received neoadjuvant chemotherapy, most of the patient in this study, except for two, were resectable without requiring preceding anticancer treatment. The impact of elevated LFT in such patients is unclear. To the best of our knowledge, there is no study that has systematically investigated the impact of elevated LFT in patients with early PDAC eligible for surgery. Given that the elevated LFT in pancreatic head cancer is a marker of bile duct invasion, ${ }^{27}$ it is assumed extent of cancer beyond the pathological staging affects the prognosis. Further studies with a larger number of patients are warranted to validate this result.

This study has some limitations. First, the design of the study was retrospective. Retrospective studies are more susceptible to selection bias. ${ }^{28}$ However, our study involved 12 referral hospitals in Korea, which should limit biases in the patients' characteristics. Second, CT protocols such as slice thickness were not standardized due to the multicenter study design. As the spatial resolution of CT is influenced by slice thickness, ${ }^{29}$ the patient's characteristics may be different if the CT protocols of the enrolled hospitals were standardized. Third, diagnostic accuracy of EUS for vascular invasion could not be evaluated due to retrospective study design. Finally, our study only included patients with masses detected by EUS. As EUS remains largely operator-dependen $\mathrm{t}^{30}$ and the likelihood of a false-negative may increase in patients with chronic pancreatitis, diffusetype carcinoma, or a recent episode of acute pancreatitis, ${ }^{31}$ the characteristics and prognosis of patients with PDAC whose masses were not found on both CT and EUS were not compared. Therefore, further studies will be necessary to investigate the role of EUS in diagnosing PDAC in patients with the aforementioned diseases.

To the best of our knowledge, this is the first study to establish the pathological stages, long-term prognosis and predictors of survival in patients with PDAC diagnosed by EUS but indeterminate on CT. The patients in the study demonstrated a favorable prognosis compared with the previously reported prognosis of patients with PDAC, which may be attributed to the detection of tumors by EUS in the early stage. EUS should be considered for patients with suspected PDAC but with indeterminate CT findings, as early detection of PDAC by EUS is associated with favorable survival.

\section{CONFLICTS OF INTEREST}

C.M.C. and G.H.K. are editorial board members of the journal but were not involved in the peer reviewer selection, evaluation, or decision process of this article. No other potential conflicts of interest relevant to this article were reported.

\section{ACKNOWLEDGEMENTS}

This study was supported by a grant from the Korean Gastrointestinal Endoscopy Research Foundation (2020 Investigation Grant).

\section{AUTHOR CONTRIBUTIONS}

Study concept and design: T.H.K., T.J.S. Acquisition of data, analysis, and interpretation of data: S.W.K., T.J.S., D.W.S., J.H.Y., C.M.C., J.H. Cho, J.H. Choi, D.W.L., S.H.L., S.B.Y., S.H.K., H.J.C., G.H.K., T.H.L. Drafting of manuscript: S.W.K. Critical revision of the manuscript for important intellectual content: T.H.K., T.J.S. Statistical analysis: S.W.K., T.J.S. Supervision: T.H.K., T.J.S. All authors read and approved the final manuscript.

\section{ORCID}

Sung Woo Ko

Tae Hyeon Kim

Tae Jun Song https://orcid.org/0000-0002-9629-5784 https://orcid.org/0000-0002-9723-2136 https://orcid.org/0000-0002-6156-8746 
Seong-Hun Kim https://orcid.org/0000-0002-7592-8060

Dong-Wan Seo https://orcid.org/0000-0001-7525-8151

Jai Hoon Yoon https://orcid.org/0000-0003-3194-5149

Chang Min Cho https://orcid.org/0000-0002-9903-1282

Jae Hee Cho https://orcid.org/0000-0003-4174-0091

Jun-Ho Choi https://orcid.org/0000-0002-8792-6249

Dong Wook Lee https://orcid.org/0000-0002-1029-9064

Sang Hyub Lee https://orcid.org/0000-0003-2174-9726

Seung Bae Yoon https://orcid.org/0000-0002-6119-7236

Tae Hoon Lee https://orcid.org/0000-0002-3545-9183

Gwang Ha Kim https://orcid.org/0000-0001-9721-5734

Hoon Jai Chun https://orcid.org/0000-0002-5539-361X

\section{REFERENCES}

1. National Cancer Institute. Surveillance, Epidemiology, and End Results Program. Cancer stat facts: pancreatic cancer, cancer statistics [Internet]. Bethesda: National Cancer Institute [cited 2019 Oct 1]. Available from: https://seer.cancer. gov/statfacts/html/pancreas.html.

2. Hart PA. Early detection of pancreatic cancer in high-risk individuals: where do we go from here? Am J Gastroenterol 2019;114:560-561.

3. Wray CJ, Ahmad SA, Matthews JB, Lowy AM. Surgery for pancreatic cancer: recent controversies and current practice. Gastroenterology 2005;128:1626-1641.

4. Hanada K, Okazaki A, Hirano N, et al. Diagnostic strategies for early pancreatic cancer. J Gastroenterol 2015;50:147-154.

5. Kitano M, Yoshida T, Itonaga M, Tamura T, Hatamaru K, Yamashita Y. Impact of endoscopic ultrasonography on diagnosis of pancreatic cancer. J Gastroenterol 2019;54:19-32.

6. Edge SB, Compton CC. The American Joint Committee on Cancer: the 7th edition of the AJCC cancer staging manual and the future of TNM. Ann Surg Oncol 2010;17:1471-1474.

7. Müller MF, Meyenberger C, Bertschinger P, Schaer R, Marincek B. Pancreatic tumors: evaluation with endoscopic US, CT, and MR imaging. Radiology 1994;190:745-751.

8. Lee ES, Lee JM. Imaging diagnosis of pancreatic cancer: a state-of-the-art review. World J Gastroenterol 2014;20:78647877.

9. Pietryga JA, Morgan DE. Imaging preoperatively for pancreatic adenocarcinoma. J Gastrointest Oncol 2015;6:343-357.

10. Natinal Comprehensive Cancer Network (NCCN). NCCN Clinical Practice Guidelines in Oncology (NCCN Guidelines). Pancreatic adenocarcinoma version2.2019 [Internet]. Plymouth Meeting: NCCN; c2019 [cited 2019 Oct 1]. Available from: https://www.nccn.org/guidelines/category_1.

11. Prokesch RW, Chow LC, Beaulieu CF, Bammer R, Jeffrey RB Jr. Isoattenuating pancreatic adenocarcinoma at multi-detector row CT: secondary signs. Radiology 2002;224:764-768.
12. Yoon SH, Lee JM, Cho JY, et al. Small ( $\leq 20 \mathrm{~mm}$ ) pancreatic adenocarcinomas: analysis of enhancement patterns and secondary signs with multiphasic multidetector CT. Radiology 2011;259:442-452.

13. Sakamoto H, Kitano M, Suetomi Y, Maekawa K, Takeyama Y, Kudo M. Utility of contrast-enhanced endoscopic ultrasonography for diagnosis of small pancreatic carcinomas. Ultrasound Med Biol 2008;34:525-532.

14. Klapman JB, Chang KJ, Lee JG, Nguyen P. Negative predictive value of endoscopic ultrasound in a large series of patients with a clinical suspicion of pancreatic cancer. Am J Gastroenterol 2005;100:2658-2661.

15. Yamaguchi K, Okusaka T, Shimizu K, et al. Clinical Practice Guidelines for Pancreatic Cancer 2016 from the Japan Pancreas Society: a synopsis. Pancreas 2017;46:595-604.

16. Deerenberg EB, Poley JW, Hermans JJ, Ganesh S, van der Harst E, van Eijck CH. Role of endoscopic ultrasonography in patients suspected of pancreatic cancer with negative helical MDCT scan. Dig Surg 2011;28:398-403.

17. Meijer OL, Weersma RK, van der Jagt EJ, van Dullemen HM. Endoscopic ultrasonography in suspected pancreatic malignancy and indecisive CT. Neth J Med 2010;68:360-364.

18. Wang W, Shpaner A, Krishna SG, et al. Use of EUS-FNA in diagnosing pancreatic neoplasm without a definitive mass on CT. Gastrointest Endosc 2013;78:73-80.

19. Srisajjakul S, Prapaisilp P, Bangchokdee S. CT and MR features that can help to differentiate between focal chronic pancreatitis and pancreatic cancer. Radiol Med 2020;125:356-364.

20. Tanaka S, Nakaizumi A, Ioka T, et al. Main pancreatic duct dilatation: a sign of high risk for pancreatic cancer. Jpn J Clin Oncol 2002;32:407-411.

21. Tanaka S, Nakao M, Ioka T, et al. Slight dilatation of the main pancreatic duct and presence of pancreatic cysts as predictive signs of pancreatic cancer: a prospective study. Radiology 2010;254:965-972.

22. Fujisawa T, Isayama H, Gunji T, Sato H, Matsuhashi N. Prevalence rate and predictive factors of pancreatic diseases in cases with pancreatic duct dilatation: a cross-sectional study of a large, healthy Japanese population. Intern Med 2020;59:769-777.

23. Yamamoto Y, Ikoma H, Morimura R, et al. The clinical impact of the lymph node ratio as a prognostic factor after resection of pancreatic cancer. Anticancer Res 2014;34:23892394.

24. Pawlik TM, Gleisner AL, Cameron JL, et al. Prognostic relevance of lymph node ratio following pancreaticoduodenectomy for pancreatic cancer. Surgery 2007;141:610-618.

25. Rau BM, Moritz K, Schuschan S, Alsfasser G, Prall F, Klar E. $\mathrm{R} 1$ resection in pancreatic cancer has significant impact on long-term outcome in standardized pathology modified for 
routine use. Surgery 2012;152(3 Suppl 1):S103-S111.

26. Darnell EP, Wang TJ, Lumish MA, et al. Preoperative cholangitis is an independent risk factor for mortality in patients after pancreatoduodenectomy for pancreatic cancer. Am J Surg 2021;221:134-140.

27. Decker C, Christein JD, Phadnis MA, Wilcox CM, Varadarajulu $S$. Biliary metal stents are superior to plastic stents for preoperative biliary decompression in pancreatic cancer. Surg Endosc 2011;25:2364-2367.

28. Geneletti S, Richardson S, Best N. Adjusting for selection bias in retrospective, case-control studies. Biostatistics 2009;10:17-31.
29. Monnin P, Sfameni N, Gianoli A, Ding S. Optimal slice thickness for object detection with longitudinal partial volume effects in computed tomography. J Appl Clin Med Phys 2017;18:251-259.

30. Hewitt MJ, McPhail MJ, Possamai L, Dhar A, Vlavianos P, Monahan KJ. EUS-guided FNA for diagnosis of solid pancreatic neoplasms: a meta-analysis. Gastrointest Endosc 2012;75:319-331.

31. Bhutani MS, Gress FG, Giovannini M, et al. The no endosonographic detection of tumor (NEST) study: a case series of pancreatic cancers missed on endoscopic ultrasonography. Endoscopy 2004;36:385-389. 